\title{
Nonlinear Dynamics of Silk and Mylar Flags Flapping in Axial Flow
}

\author{
Hamid Ait Abderrahmane', Michael P. Païdoussis ${ }^{1}$, Mohamed Fayed ${ }^{2}$, Hoi Dick Ng² \\ ${ }^{1}$ Department of Mechanical Engineering, McGill University, 817Sherbrooke St. W., Montréal, Québec, H3A 2K6 Canada \\ ${ }^{2}$ Department of Mechanical and Industrial Engineering, Concordia University, Montréal, Québec, H3G 1 M8 Canada
}

\begin{abstract}
In this study, experiments were conducted on the flapping dynamics of flags immersed in an axial flow generated by a laboratory scale wind tunnel. The experimental setup was first validated by comparing the critical conditions for flapping onset with relevant results from experimental investigations, numerical simulations and theoretical models found in the literature. Short and long flags, as well as flags with aspect ratio equal to unity, were considered. Nonlinear dynamic analyses including Power spectra, phase-space portraits, Poincaré sections and circle maps obtained from the flag transverse displacement time-series indicate that the observed non-periodic flapping mainly arises through a quasi-periodic scenario. This paper also reports for the first time a "switching" oscillation phenomenon, which is closely related to hysteresis and bistability phenomena.
\end{abstract}




\section{Introduction}

The flapping of a flexible flag immersed in an axial flow is a canonical problem in fluidstructure interactions. It is used as a model-example to understand many real-world problems of fluid-structure interactions in science, engineering and biology. For comprehensive reviews, the reader is referred to Païdoussis [1] and Shelley and Zhang [2].

Flapping of a flexible plate or flag immersed in an axial flow is a self-sustained phenomenon; it involves the coupled dynamics of the fluid flow and the flexible plate. The fluid dynamics involves a destabilizing pressure jump across the deflected plate and vorticity shedding at the trailing edge. The plate dynamics includes the restoring forces due to the bending rigidity, structural damping, and the flow-induced variable tension along the plate. A transfer of energy between the fluid and the flag leads to a complex dynamical behavior, resulting either in the stationary stretched-straight state, or in periodic and chaotic flappings. A full understanding of the flapping dynamics remains elusive, and this justifies the extensive recent theoretical, numerical and experimental studies on the subject; see, for instance, Argentina and Mahadevan [3], Shelley, Vandenberghe and Zhang [4], Alben and Shelley [5,] Michelin et al. [6], Zhu and Peskin [7,8], Connell and Yue [9], Tang and Païdoussis [10] and Eloy et al. $[11,12]$.

Theoretical approaches for the analysis of the flapping of a flexible plate immersed in axial flow adopt a number of hypotheses. For instance, Shelley et al. [5] derived a simple relation for the critical flow speed at which the instability (flapping) sets in, by assuming an infinite plate in both directions; then modeling the plate as an Euler-Bernoulli beam and assuming the fluid flow to be a potential flow. However, in practice, flexible plates are of finite length and width; both dimensions affect the stability conditions of the plate. The studies dedicated to the influence of length and width 
on the flapping dynamics may be divided into two categories, corresponding to the two asymptotic limits of the width-to-length ratio $H / L$, i.e., the aspect ratio. When the aspect ratio is small the plate may be considered to be a strip, and the problem can be modeled using the slender-body approximation and an unsteady potential flow model; see Datta and Gottenberg [13] and Lemaitre et al. [14]. In the latter it was shown that the critical flow velocity at which the flapping starts depends on the length of the strip. Lemaitre et al. [14] reported that for short strips the critical flow velocity depends on the length, whereas it becomes independent of the length for longer strips. When the aspect ratio is very large or infinite, the problem may be considered as being two-dimensional and the more physical models take into account vortex shedding (wake) at the trailing edge. Kornecki et al. [15] were the first to use this two-dimensional approach to study plates in axial flow. The plate was modeled as a linear beam and the aerodynamics at the trailing edge was modeled by the Kutta condition (Theodorsen [16]) which ensures vortex advection. Later, Shayo [17] extended the work of Kornecki et al. [15] and considered plates of finite width. It was concluded that increasing the aspect ratio, i.e., the width of the plate, destabilizes the plate. This conclusion appears to be incorrect according to the recent studies of Eloy et al. [11, 12], who investigated the influence of the aspect ratio on the instability condition. They discussed the discrepancy between experimental and theoretical (two-dimensional model) predictions on the critical condition for instability. As pointed out by Eloy et al. [11,12], the two-dimensional models of Kornecki et al. [15], Huang [18], Watanabe et al. [19] and Guo and Païdoussis [20] predict instability of the plate at much lower flow velocities than observed in experiments. Eloy et al. $[11,12]$ argued that the two-dimensional limit cannot be achieved experimentally because of the hysteresis phenomenon and three-dimensional effects inherent in flutter of large aspect ratio plates. Also, Tang et al. [21] studied the energy transfer from the fluid to the plate (and vice versa) and proposed a scheme for its exploitation for power generation. 
The problem of a flapping flexible plate in axial flow was also investigated numerically. Two common techniques were used: the first approach utilized immersed boundary methods (Zhu and Peskin [6]) and the second coupled fluid-solid solvers (Connell \& Yue [9]). Zhu \& Peskin [6] used the immersed boundary method to simulate the soap film flow experiments conducted by Zhang et al. [22]. Similar dynamics and vortical structures found in the experiment were retrieved. Connell \& Yue [9] investigated the flapping dynamics of a thin two-dimensional flag of high extensional rigidity and low bending rigidity. They conducted a fluid-structure direct simulation (FSDS) that couples a direct numerical simulation of the Navier-Stokes equations to a solver for thin-membrane dynamics of arbitrarily large amplitudes. They found that the response of the thin membrane depends on the mass ratio and they identified three distinct regimes: (i) the first one is a stationary solution corresponding to fixed point response, observed for small mass ratios; (ii) the second is a period-one limit-cycle flapping for intermediate values of the mass ratio; and (iii) the third is a chaotic flapping regime found for large values of the mass ratio.

The flapping of a flag in axial flow has also been extensively studied experimentally. In contrast to both theoretical and numerical investigations which are based on assumptions that simplify the problem, the experimental approach is free of such restrictive assumptions. Another import issue which is difficult to properly handle in both theoretical modeling and numerical simulation is the initial planeity defect. In fact, in the absence of, or weak, wind flow the flag tends to bend, sag and twist. In the case of flag made of soft material the flag can also fold in many layers. This justifies the extensive experimental investigations aiming to provide new insights into the phenomenon and to validate the different theoretical and numerical models. Several experimental studies may be found in the recent literature. Among these studies, one should refer to the experiments performed by Zhang et al. [22], Shelley et al. [4] Watanabe [19] and Eloy et al. [12]. Zhang and his co-authors investigated the flapping of a flexible slender filament in a soap-film 
tunnel. Shelley et al. investigated the flapping of a flexible plate in a water tunnel, while the experiments by Watanabe [19] and Eloy et al. [12] were in a wind tunnel. Note that the flapping of a thin filament in a soap-film tunnel can be considered as a two-dimensional problem, which is a hypothesis difficult to achieve in wind and water tunnel experiments where the width of the flag plays an important role in the stability of the plate. The majority of the experimental studies on flapping flag has focused on the critical stability conditions, i.e., the condition at which a flag starts flapping. To the best knowledge of the authors, the literature on experimental analysis of the flag flapping regimes is inexistent or scarce.

The present work is an experimental contribution that adds to the wealth of experimental data on the flapping of flags. Previous experiments in literature revealed the importance of a number of parameters such as the length-to-width and mass ratios, and the bending rigidity of the plates. Moreover, besides the structural and aerodynamic forces a flag dynamics includes also complex oblique wave patterns travelling along the flag surface, which can be easily observed on flapping flag in windy day. These waves should originate from the initial position of the flag in the absence of any wind. Such oblique waves start with relatively large amplitude; as it will be argued below these may explain the nonlinear character of the dynamics of flapping flags.

This paper is organized as follows. First we describe the validation of our experimental setup through the investigation of the critical instability conditions, i.e., the flow speed at which a bifurcation arises from a stationary to a fluttering state. In the second step, we explore the flapping dynamics based on reconstructions of the attractors from experimental data. These attractors and the transitions between them are discussed using tools from nonlinear time-series analysis, such as the correlation dimension, Lyapunov exponents, Poincaré sections and circle maps. In last section, the discussion of the results is summarized and concluded. 


\section{Experimental setup}

\subsection{Description of the setup and flutter threshold}

To investigate the rich dynamics at work in the flutter phenomenon we used a closed-loop wind tunnel (see Fig.1). This is relatively high turbulence intensity tunnel with mean velocity variations of $\Gamma= \pm 3 \%$, so that the flag therein may be considered to be subject to continual perturbations*. The test-section is rectangular, $0.45 \mathrm{~m}$ high by $0.25 \mathrm{~m}$ wide, and it is $1.5 \mathrm{~m}$ long. Transparent walls make observation easy. The flow speed, measured by a Pitot tube was increased from 0 up to approximately $6 \mathrm{~m} / \mathrm{s}$ in an increment of $0.5 \mathrm{~m} / \mathrm{s}$. The flags were made of silk or Mylar of different span width $H$ and length $L$. The flags were aligned with the flow in the wind tunnel, and were attached to the trailing edge of a $0.45 \mathrm{~m}$ long NACA0010 airfoil section to ensure uniform flow along the flag. The Mylar sheet used in the experiments has the following properties: density of 1390 $\mathrm{kg} / \mathrm{m}^{3}$, Young's modulus $E=5.17110^{9} \mathrm{~N} / \mathrm{m}^{2}$, thickness $h=0.0765 \mathrm{~mm}$ and Poisson's ratio $v=$ 0.38 ; the flexural rigidity was determined using the relation $D=E h^{3} / 12\left(1-v^{2}\right)$. The silk sheet has the following properties: density of $563 \mathrm{~kg} / \mathrm{m}^{3}$, and thickness $h=0.23 \mathrm{~mm}$; its flexural rigidity was estimated using the following relation (Timoshenko [23]): $D=h_{c}^{3} m g / 7.83$, where $m$ is the mass per unit area and $h_{c}$ is the minimum height for which a vertical strip clamped at its bottom buckles under its own weight.

In the experiments, motion of the flapping plate was recorded with a high-speed CMOS video camera (PCO.1200hs) from above, through the top wall of the wind tunnel, at a rate of 60 images per second; the camera was aligned with the top edge of the plate. Afterwards, the images

*This, in the context of this paper, is considered as a positive feature; by comparing the results to those obtained in very low-turbulence wind tunnels, we can assess how strongly the results are affected by turbulence. 
were processed and the displacements (amplitudes) of three different points on the plates, located $1 / 4,2 / 4$ and $3 / 4$ of their length, were extracted.

Similar to other experiments, we have observed three main regimes. The first one is a stationary regime; this was observed at small flow velocities. In this regime the Mylar or silk flag was aligned with the flow and remained stable, until the critical flow velocity $U_{c}$ was reached. Above the critical flow velocity the structure started to oscillate (flutter) spontaneously; this is the second regime. At higher flow speeds, the flapping dynamics becomes more and more complex; this last regime is referred to as chaotic flapping. The experimental procedure is as follows. First, we set the flow velocity at a small value, at which the plate remained stationary. We then increased the flow velocity gradually in a $0.5 \mathrm{~m} / \mathrm{s}$ increment to capture the critical value, i.e., the value at which the structure would start flapping. The flow velocity was then increased further, with the same increment, up to the limit allowed by the experimental setup $(6 \mathrm{~m} / \mathrm{s})$. Afterwards, the flow velocity was decreased, with the same steps as before, until the plate returned to its initial stable state, which defined a second critical speed. Similar to previous experiments (e.g., Eloy [12]), in some cases the descending critical speed is much lower than the ascending one, indicating the existence of a hysteresis. This phenomenon was observed when the flexible plate is initially subject to a small outof-plane bend under its own weight, rather than being perfectly vertical; this occurs for low bending rigidity or for elongated (low aspect ratio) flags.

The dynamics of the flapping regime was investigated through nonlinear time-series analysis technique of the amplitudes of the three aforementioned points on the flexible plate. No significant difference in the dynamics was noticed; hence, in what follows, the dynamics of the flapping is discussed using only the data collected for the measuring point at $3 / 4 \mathrm{~L}$. The technique was proposed by Packard et al. [24]. It consists of the reconstruction of a multi-dimensional phase portrait, which reveals much about the dynamical behavior of the system, from measurements of a 
single variable. The procedure relies on the reconstruction of the whole system trajectory in an "embedding space", using the delay-time method of Takens and Mañé [25]. A selection of appropriate values for "embedding space" and delay-time is particularly crucial for the reliability of the results. To ensure equivalence between the topological properties of the actual and reconstructed attractors we adopted the interactive technique suggested by Albano et al. [26]. The authors have combined a singular-value decomposition, which leads to a set of statistically independent variables, and the wellknown Grassberger-Procaccia algorithm [27] to determine the dimension of the attractors, which is particularly useful and can be used to differentiate between deterministic and stochastic chaos.

In the first set of experiments, measurements were made of the critical flow velocities at which the flag starts flapping and the corresponding frequencies. As mentioned earlier, in the wind tunnel used in the experiments the turbulence intensity $(\Gamma)$ is relatively high. Indeed, in the wind tunnels used in some of the previous experiments discussed in the introduction, including those in soap-film tunnels (e.g., Zang et al. [21] and Ait Abderrahmane et al. [28]), $\Gamma$ was very low in some cases less than $1 \%$. If, as expected, the effect of the higher $\Gamma$ does not fundamentally change the dynamics qualitatively (and not very much quantitatively either), then these experiments can serve to validate the experimental setup and methods of data analysis.

The critical velocity is plotted as a function of the mass ratio $\left(\mu=\rho_{f} L / \rho_{m} h\right)$ for the Mylar and silk flags in Fig.2; $\rho_{f}$ is the fluid density, $\rho_{m}$ is the flag density and $h$ is the flag thickness. The results are compared with the theoretical models of Yamaguchi et al. [29], Tang and Paidoussis [10], Eloy et al. [12], Kornecki et al., [15] and Michelin et al. [6].

The curves in Fig. 2 represent the theoretical critical velocities above which the flag becomes unstable and starts to flutter. The first observation is that our experimental results for the Mylar flags are fairly close to those predicted by the theoretical models. The second remark is that the critical 
flow velocity increases with the width of the structure. In other words, increasing the width of the flag has a stabilizing effect. This can be explained by the fact that a flag with large width can bend in the span-wise direction which would tend to stiffen the structure and delay the instability. Similarly, the initial bending, sagging and twisting of the silk flags (soft material) can explain why they appear to be more stable than the Mylar flags as well as the theoretical prediction. Another cause of the higher stability of silk flags may be the in-plane deflection, flow-induced tension that tends to reduce the initial deformation that might trigger flapping. This initial deflection of the structure could also be responsible for the hysteresis phenomenon.

Another test of our system is in terms of the flapping frequency at the critical conditions, i.e., the frequency at which the flag starts to flap, which is determined using power spectral analysis. These frequencies are compared to those predicted by the model of Tang and Païdoussis [10] in Fig.3, showing that this model predicts fairly well the initial flapping frequency.

Based on the discussion above, we can state that despite the relatively high turbulence in the wind-tunnel, our experimental tests follow well those predicted by theory and low- $\Gamma$ experiments. It seems therefore that the perturbations implicit in higher turbulence flow within the wind-tunnel does not affect the critical conditions for the flapping.

\section{Results of the dynamical analysis of the flapping state}

In this section we analyze the onset of flapping and its transition to a chaotic regime using a nonlinear time-series analysis method. This method consists of the reconstruction, from the transverse displacement time series of the flag, of the attractors that describe the nonlinear dynamics of the flapping in phase space. Both the geometry of the attractors and the stability of orbital 
trajectories on it are discussed. It is worth noting that the time series is first de-noised, using the empirical mode decomposition technique of Huang et al. [30]. This method consists of dissecting the raw time-series into its intrinsic modes. Only the modes with significant amplitudes are considered; the others, with small amplitudes, are considered as intrinsic noise of the time series and they are neglected. Based on the discussion above on the influence of the aspect ratio on the critical condition for the onset of flapping, one should expect that the aspect ratio influences the flapping dynamics.

In what follows, we separate the discussion onto three: for short flags, long flags and flags of aspect ratio 1 . This is rather arbitrary, since our long flags are not very long $(H / L=2$ and $H / L=1.67$ for Mylar and silk flags, respectively) nor our short flags very short $(H / L=0.1$ and $H / L=0.47$ for Mylar and silk flags, respectively). Nevertheless, this division is for convenience of the discussion.

\subsection{Short flags}

The results of the nonlinear analysis of the onset of flapping of a short Mylar and a short silk flag are displayed in Fig.4. The de-noised time series of the transverse displacement of a point at the top edge of the flag located at 3/4 from the leading edge is shown in Fig. 4(a) and (b) for Mylar and silk flags, respectively. Both time series show erratic oscillations, while the corresponding power spectra displayed in Fig. 4(c) and (d) indicate that the onset of flapping of short Mylar and silk flags seems to be different. Fig.4(c) shows several peaks clustered around two incommensurate frequencies, $f_{1}=16.64 \mathrm{~Hz}$ and $f_{2}=26.6 \mathrm{~Hz}$, which may indicate that the flapping starts as a chaotic oscillation. The second frequency, $f_{2}$, is approximately $3 / 2$ times of the fundamental one, $f_{1}$. The presence of such a frequency in the flapping power spectra was also reported by Connell and Yue [9]. They indicated that the presence of this frequency is a prelude for chaotic flapping. This second frequency should be related to the oblique wrinkles or waves travelling along the surface of the flag. 
The power spectrum for the short silk flag in Fig. 4(d) indicates that flapping is dominated by one fundamental frequency and its harmonics. The flapping dynamics of the silk flag, which has a flexural rigidity ten times smaller than of a Mylar flag, appears to be more regular that the one of Mylar flag. The regularity of silk flag flapping could be explained by the fact that tension force, induced from the fluid drag, strongly stretched the silk flag and alleviated the amplitude of the oscillatory motion due to oblique travelling waves. The reconstructed attractor from the time series for the Mylar flag is shown in Fig. 4(e). Three different time intervals are depicted with the aid of different colors; the orbital trajectories are wrinkled in a complicated way around a "tire-like" attractor. The correlation dimension of the Mylar flag attractor is estimated, using the standard Grassberger-Procaccia algorithm; its value is approximately $D=6.5$ which indicates that the geometry of the attractor is "strange". The sensitivity to the initial conditions of the trajectories on the "tire-like" attractor is verified through the estimation of the Lyapunov exponents; the exponents were calculated using the statistical approach proposed by Lai and Chen [31]. The largest positive Lyapunov exponent is positive and its value is 0.05 ; this means that, along the direction that corresponds to this exponent, the orbital trajectories diverge from each other with time; the dynamics around the tire-like attractor can be qualified as weakly chaotic. The dynamics on the "tire-like" attractor can be viewed as two complicated oscillations that can be described by two cyclic coordinates. Sectioning the "tire-like" attractor with a plane, transverse to long cycle coordinate, one can obtain the Poincare section of the attractor shown in Fig. 4(g). This section presents a ring-like structure where lines connecting successive points of the Poincaré section often cross the central hole of the ring-like structure, which is evidence that the flapping is chaotic. The ring-like structure indicates that the observed chaos has arisen from quasi-periodic regime; see Nayfeh and Balachandran [36].

A similar, tire-like strange attractor and ring-like structures in the Poincaré section were observed in the case of silk flag; see Fig. 4(f) and (h). The onset of silk flag flapping is also chaotic and also 
seems to arise via quasi-periodicity. The correlation dimension of the attractor and the largest Lyapunov exponent were estimated and found equal to 5.6 and 0.03 , respectively.

The onset of flapping in a weakly chaotic regime for both Mylar and silk flags can also be confirmed through the corresponding circle maps, see Fig.4 (i) and (j), plotted as follows: first we find a centroid of the points that form the Poincaré section and consider $\theta_{\mathrm{n}}$ to be the polar angle that the $n^{\text {th }}$ point makes with the horizontal axis passing through the centroid. The circle map is a plot of $\theta_{\mathrm{n}}$ against $\theta_{\mathrm{n}+1}$; it records the progression of the state points on the Poincaré section. Cloudy figures in the two circle maps (- Fig.4 (i) and (j) indicate that the dynamics on the tire-like attractor in Fig.4(e) and (f)) are in fact chaotic.

According to the present results, it appears that, for both the short Mylar and silk flags, the onset of flapping is chaotic via the quasi-periodic route. This quasi-periodicity is explained by the interplay of the pure flapping oscillations and the flags oscillations that results from the oblique travelling waves on the flag surface. The second oscillation modulates the fundamental oscillations (flapping). The fundamental frequency is the one of the flapping oscillation; this frequency should be related to the one at which vortices are shed from the flag trailing edge. The amplitude of the oblique waves, which the wind flow transports from the leading towards the trailing edge, is of relatively small amplitude (typically two orders of magnitude less than the flapping amplitude (see Fig.4c) for Mylar. The amplitude of these waves can be relatively small that they cannot be captured with aide of power spectrum analysis; see flag (Fig.4d) for silk flag. In fact, short flags start flapping at relatively high flow speed $(3 \mathrm{~m} / \mathrm{s})$ which induces strong tension force and alleviate the ondulation of the surface of the flag, in particular in silk flag. Equivalently, it is worth noting that the onset of chaotic flapping through quasi-periodic route was also observed in flapping of flexible filament in soap film tunnel see [28]. 


\subsection{Flags with aspect ratio equal to unity}

Similar analysis was conducted with Mylar and silk flags with aspect ratio equal to unity, i.e., flags having equal length and height. The reconstructed attractors and correlation functions for the Mylar and silk flags are shown in Fig. 5.

The projection of the reconstructed attractor in three-dimensional space for the Mylar flag is depicted in Fig. 5(a). It appears that the attractor is formed from two tori, one embedded in the other. The trajectories on the attractor are drawn with three different colors for three consecutive time intervals. In contrast to the short flag the flapping dynamics seems to be less irregular. The correlation dimension of this attractor is estimated to be $D=5.6$; the attractor is strange. The divergence of the orbits on this attractor is investigated through the Lyapunov exponents; the largest Lyapunov exponent is 0.05 which indicates that the flapping is weakly chaotic. Thus, from onset, the flapping of the Mylar flag is chaotic. This character of the flapping can also be discerned through the power spectrum (not shown here) which indicates one fundamental frequency and a continuous decrease of energy beyond that. A Poincaré section, Fig.5(c), shows that the line connecting successive points is broken; moreover, instead of moving forward, in some instances the points move backwards, which indicates that for chaotic flapping of the Mylar flag the attractor is no longer associated with a torus. Such non-monotonic evolution on the attractor is also seen on the circle map shown in Fig.5 (e), and such behavior is associated with a torus breakdown scenario; see Nayfeh and Balachandran [36 ]. Hence, one could infer that the chaotic flapping of the Mylar flag might arise through torus breakdown, present in many natural and human-made systems. The chaotic dynamics, issued from the torus breakdown, are characterized by the strange attractor with small positive Lyapunov exponent, as is the case of the Mylar flag. The projection in 3-D space of the 
reconstructed attractor for the silk flag is shown in Fig.5 (b). The attractor seems to be made up of a folded torus. The estimated correlation dimension is a non-integer number, equal to 4.8. The largest Lyapunov exponent is positive and equal to 0.01 ; because of the remaining noise in the time series, one might consider this small value of the largest exponent to be zero and infer thereby that the dynamics on the attractor in Fig. 5(b) is not chaotic. In fact, the corresponding Poincare section of the attractor in Fig. 5(d) shows that the intersection point precesses around a closed curve in a monotonic way. This monotonic dynamics can also be seen through the circle map shown in Fig. 5(f). It mimics the dynamics on a torus. Hence, the attractor in Fig. 5(b) can be considered as a torus in a high embedding dimension or a strange non-chaotic attractor because of its fractal correlation dimension. Such attractors are ubiquitous in quasi-periodic forced physical systems; see Grebogi et al. [32] and Ditto et al. [33]. Because of a relatively high level of the flow fluctuations in our wind tunnel, such quasi-periodic forcing of a flag is probable.

Continuing with the silk flag, and increasing the flow speed, the attractor is of "tire-like" form; see Fig. 6(a). The largest Lyapunov exponent increases and is found to be equal to 0.4; thus, the flapping is now chaotic. This chaotic state is confirmed through a Poincaré section which has the form of a thin ring on which the Poincaré point marches in a circular path. This ring-like form indicates that the chaotic dynamics on the attractor has emerged via the quasiperiodic route to chaos. The power spectrum of the flapping shown in Fig. 6(c) indicates the presence of a $3 / 2$ harmonic of the fundamental frequency, identified by Connell and Yue [9] as an indication of chaotic flapping. With further increase in flow speed, the flapping dynamics becomes even more complicated; in fact the Poincaré section loses its ring-like structure and becomes a cloud of points; see Fig. 6(d).

The results show that a Mylar flag starts flapping directly in chaotic state; this state appears to be issued through a torus breakdown scenario. However, for silk flag the transition towards chaotic flapping followed a quasi-perdiodic route as in the case of short flags. According to the results above 
and also later the results in the following section, the transition towards a chaotic flapping through torus breakdown seems to be singular. Note that in the chaotic state, the dynamic could be sensible to the initial conditions, e.g., the Mylar flag sags under its weight while the silk flag wrinkles in many folds. It is suggested that the torus breakdown scenario should be confirmed through further experimental investigations.

\subsubsection{Long flags}

We pursue our investigation, now considering the flapping of long flags, i.e., flags with length higher than the height $(H / L=0.1$ and $H / L=0.47$ for Mylar and silk flags, respectively). The results for both Mylar and silk flags are summarized in Fig .7. The 3-D projections of the reconstructed attractors are shown in Fig. 7(a) and (b) for Mylar and silk flags, respectively. The attractor in Fig. 7(a) forms a twisted torus; the correlation dimension is approximately $D=4.2$ and the estimated largest Lyapunov exponent is null. Hence, the onset of flapping for the long Mylar flag is not chaotic and the fractal dimension of the attractor suggests that the attractor it is a strange nonchaotic attractor. In fact, the Poincare plot in Fig. 7(c) indicates that the points describe a closed circular curve which is a characteristic of a torus attractor. Moreover, the corresponding circle map, Fig. 7(e), indicates a monotonic evolution of the Poincaré points on a closed curve, which confirms that the flapping trajectories lie on the torus-like attractor, i.e., the flapping at its onset is quasi-periodic.

The onset of flapping of a long silk flag appears to be chaotic. In fact, the reconstructed attractor is of tire-like form; the Poincare plot exhibits a ring like structure but the lines connecting subsequent states cross the empty core region; see Fig. 7(b) and (d). This indicates that the flapping onset is chaotic and similarly to the cases discussed above, the transition should be through a quasi- 
periodic route. The corresponding circle map, Fig. 7(f), indicates a cluster of points around four regions located on the first bisection line; this indicates that the flapping involves at least four distinct modes and the points far from the first bisection are associated with the transitions between the involved modes. Note that such a circle map is characteristic of chaotic dynamics.

Increasing the flow speed from $1.84 \mathrm{~m} / \mathrm{s}$, at which the flapping of the long Mylar flag starts, to $3.3 \mathrm{~m} / \mathrm{s}$, the attractor forms a tire-like figure (Fig. 8(a)). The Poincaré section and its corresponding circle map indicate that the flapping has become chaotic. It seems that this chaotic flapping has arisen from the quasi-periodic regime. The circle map in Fig. 8(d) indicates clusters in three different regions near the first bisection, which means that the chaotic flapping regime involves three oscillatory modes and that it is switching between these modes.

The behavior of long flags contrasts with that of flags with aspect ratio equal to unity also in this sense: the onset of flapping long Mylar flags is not chaotic, whereas that of long silk flag is. For the silk flag, the stabilizing role of the flow-induced tension seems to be overcome by the destabilization due to the decrease of the flexural rigidity which is inversely proportional to the flag length. The initial conditions also seem to play a key role in the present experiments. Because of the gravity, the flags tend to sag or fold in several wrinkles around the holding airfoil. The silk flag wrinkles in much more folds than the Mylar flag which tends more to sag. Hence, when the wind begin to blow up the initial conditions are different for the two flags. The amplitude of the oblique wave at the surface of the flag should be larger for the silk flag, while the long Mylar flag tends more to snap. The nonlinear effect should be more prominent in silk flag which triggers chaotic flapping dynamics.

As a summary of this subsection, devoted to the analysis of the dynamics of flapping flag, one can stress the fact that a flapping is in nonlinear regime generally involving two oscillatory motions. The first one should be the pure flapping of the flag in similar way as a rigid plate. The second 
oscillatory motion of amplitude relatively small in comparison with those of the flapping oscillation is due to the oblique wave that travels along the flag surface. Note that similar modulation of the flapping by a low amplitude wave was reported recently in [28] in the case of flapping filament in soap film tunnel. The main route towards chaotic flapping is a quasi-periodic route. The interaction of the pure flapping dynamics and the travelling oblique waves seems to involve several modes for long flags and the chaotic regime exists as a complex switching from one mode into another. In the case of Mylar flag with aspect ratio equal to unity, a transition towards chaos through torus breakdown is found. However, further experiments are required to confirm this new finding.

\subsection{Switching oscillation phenomenon}

In the case of the long Mylar flag, we have observed a peculiar but robust phenomenon. At a flow velocity just below the critical, the flag flaps for a certain time, and then stops for a very short time before flapping again. The dynamics seems to be a continuous back and forth between the stationary and flapping regimes; the two regimes coexist and interchange their stability. This peculiar behavior is observed when the flapping dynamics exhibits a robust hysteresis; it occurs in the bistability region where stationary and flapping solutions coexist. To illuminate this peculiar dynamics, we have used the empirical mode decomposition of the raw time-series into two modes. The raw time series of the transverse displacement of the flag is shown in Fig. 9(a). The first two intrinsic modes extracted from this time-series are depicted in Fig. 9(b) and (c). These two modes constitute the two unstable solutions: one is stationary and the second is oscillatory. The switching of the flag between these two solutions is illustrated through the corresponding attractors in phase space; see Fig. 9(d). The fixed point represents a stationary solution, while the limit-cycle represents the quasiperiodic oscillatory solutions found at the flapping onset (Fig. 7(a), (c) and (e)). This peculiar 
dynamics is observed only in the case of long flags for which the flow speed range over which hysteresis occurs is relatively large. It is worth noting that this switching-oscillation phenomenon is not accidental; it is repeatable, and it has been also observed in our laboratory in experiments with long filaments placed in a soap film tunnel that exhibit strong hysteresis [28].

\subsection{Conclusion}

In this paper, we have studied experimentally the flapping dynamics of flags in relatively high turbulent flow. We have considered two parameters that might influence the flapping dynamics, namely the flexural rigidity and aspect ratio of the flag. In fact two materials, Mylar and silk, were used; the former has a flexural rigidity approximately ten times larger than the second one. Flag aspect ratios inferior, equal and superior to one were considered. We have first shown through the validation of our experimental setup that turbulence in the flow might have but only a small effect on the onset of flapping. Furthermore, the measured instability thresholds follow the trends predicted by the theory and confirm the influence of the flag aspect ratio on the onset of the flapping. The onset condition seems to be affected by the initial bending, sagging and twisting which might explain the fact that silk flags appear to be more stable than the theoretical prediction because of the softness of the material.

Using power spectra and nonlinear time-series analysis, flapping was investigated and its different regimes were discussed using the reconstruction of the attractors from the time-series. The orbital trajectories of the flapping on the attractor were discussed using the Lyapunov exponents, Poincaré sections and circle maps. The results show that both the flexural rigidity and aspect ratio influence the flapping dynamics of the flag. 
The results may be summarized as follows. (i) both Mylar and silk short flags start to flap directly in the chaotic regime. (ii) Similar behaviour was observed for flags with aspect ratio equal to unity and made from Mylar, while those made from soft material (silk) exhibit a complicated flapping regime, but with a certain level of order within it; the flapping dynamics is described by a Strange Nonchaotic Attractor ( $S N A$ ) and such attractors were found in quasi-periodic driven systems. (iii) Long silk flags star to flap directly in the chaotic regime, while those made of Mylar start to flap first in a quasiperdiodic regime before becoming chaotic. The main result of the present study is that flapping regime is intrinsically a quasi-periodic system consisting of at least two coupled oscillatory motions: the main one is the oscillation of the flag as a rigid plate clamped at its leading edge. The second oscillation is the relatively low-amplitude and low frequency modulation of the first one due complex wave patterns of oblique travelling waves (modes) of the flag surface. Bending vibrations could also interfere with flapping and oblique waves in particular in the case of Mylar for which the bending rigidity is relatively high. The interplay of these oscillations form a quasiperiodic regime which linear analysis (power spectra) fail to capture in most of the cases. In this case the Poincaré section consists of discrete points marching on a closed curve, and the circle map describes a monotonic evolution of the Poincaré on this closed curve. When the secondary oscillatory motion involves more than one mode; the Poincaré section exhibits a ring-like structure, which is an indication of complex multifrequency interactions. A quasiperiodicity is inherently unstable and degenerates into chaos through the well known quasiperiodic scenario Nayfeh and Balachandran [36]. This ring-like structure, during the development of the flapping dynamics towards chaotic states, seems to be robust.

Finally, we report for the first time a phenomenon of switching-oscillations that consist of the back and forth alternation between two states of the flag, stretched straight and flapping states. This phenomenon is inherent in the bistability and hysteresis phenomena already reported in the literature. 
Similar to the two behaviours, the phenomenon of switching-oscillation can serve as test for nonlinear theoretical and numerical models.

\section{Acknowledgments:}

The authors gratefully acknowledge the support by the Natural Sciences and Engineering Research Council of Canada (NSERC).

\section{References}

1. Paidoussis, M.: Fluid-Structure Interactions: Slender Structures and Axial Flow, Vol. 2, chapter 10. London: Elsevier (2004)

2. Shelley, M., Zhang, J.: Flapping and bending bodies interacting with fluid flows. Annu. Rev. Fluid Mech. 43, 449-465 (2011)

3. Argentina, M., Mahadevan, L.: Fluid-flow-induced flutter of a flag. Proc. Natl. Acad. Sci. USA. 102 1829-34 (2005)

4. Shelley, M., Vandenberghe, N., Zhang, J.: Heavy flags undergo Spontaneous oscillations in flowing water. Phys. Rev. Lett. 94094302 (2005)

5. Alben, S., Shelley, M.: Flapping states of a flag in an inviscid fluid: bi-stability and the Transition to Chaos. Phys. Rev. Lett. 100074301 (2008)

6. Michelin, S., Llewellyn-Smith, S., Glover, B.: Vortex shedding model of a flapping flag. J. Fluid Mech. $6171-$ $10(2008)$

7. Zhu, L., Peskin, C.: Interaction of two flapping filaments in a flowing soap film. Phys. Fluids 15 54-60 (2003)

8. Zhu, L.D., Peskin, C.: Simulation of a flapping flexible filament in a flowing soap Film by the immersed boundary method. J. Comput. Phys. 179 452-68 (2002) 
9. Connell, B., Yue, D.: Flapping dynamics of a flag in a uniform stream. J. Fluid Mech. 581 33-68 (2007)

10. Tang, L., Païdoussis, M.P.: On the instability and the post-critical behavior of two-dimensional cantilevered flexible plates in axial flow. J. Sound Vibrat. 305 97-115 (2007)

11. Eloy, C., Lagrange, R., Souilliez, C., Schouveiler, L.: Aeroelastic instability of cantilevered flexible plates in uniform flow. J. Fluid Mech. 611 97-106 (2008)

12. Eloy, C., Souilliez, C., Schouveiler, L.: Flutter of a rectangular plate. J. Fluids Struct. 23 904-19 (2007)

13. Datta, S.K., Gottenberg, W.G.: Instability of an elastic strip hanging in an airstream. J. Appl. Mech. 42 195-198 (1975)

14. Lemaitre, C., Hemon, P., de Langre, E.: Instability of a long ribbon hanging in axial air flow. J. Fluids Struct. 20 913-925 (2005)

15. Kornecki, A., Dowell, E.H., O’Brien, J.: On the aeroelastic instability of two-dimensional panels in uniform incompressible. J. Sound Vibrat. 47 163-178 (1976)

16. Theodorsen: Theodore General Theory of Aerodynamic Instability and the Mechanism of Flutter. nacareport 496 (1935).

17. Shayo, L.K.: The stability of cantilever panels in uniform incompressible flow. J. Sound Vibrat. 68 341-350 (1980)

18. Huang, L.X.: Flutter of cantilevered plates in axial flow. J. Fluid Struct. 9 127-147 (1995)

19. Watanabe, Y., Suzuki, S., Sugihara, M., Sueoka, Y.: An experimental study of paper flutter. J. Fluids Struct. 16 529-42 (2002)

20. Guo, C.Q., Païdoussis, M.P.: Stability of rectangular plates with free side-edges in two-dimensional inviscid flow. J. Appl. Mech. 67 171-176 (2000)

21. Tang,L.,Paidoussis,M.P., Jiang,J.: Cantilevered flexible plates in axial flow: energy transfer and the concept of flutter-mill, Journal of Sound and Vibration. 326, 263-276 (2009).

22. Zhang, J., Childress, S., Libchaber, A., Shelley, M.: Flexible filaments in a flowing soap film as a model for one-dimensional flags in a two-dimensional wind. Nature 408 835-39 (2000)

23. Timoshenko, S.: Theory of Elastic Stability. McGraw-Hill, New York (1961).

24. Packard, N.H., Crutchfield, J.P., Farmer, J.D, Shaw, R.S.: Geometry from a time series. Phys. Rev. Lett. 45 712-716 (1980)

25. Takens, F., Mañé, R.: Dynamical Systems and Turbulence. Warwick, Lecture Notes in Mathematics, R. Rand and L.S. Young, ed., Springer, Berlin (1981)

26. Albano, A.M., Muench, J., Schwartz, C., Mees, A.I., Rap, P.E.: Singular-value decomposition and the Grassberger-Procaccia algorithm. Phys. Rev. A 38 3017-3026 (1988)

27. Grassberger, P, Procaccia, I.: Measuring the strangeness of strange attractors. Physica D 9 189-208 (1983) 
28 Ait Abderrahmane,H, Paidoussis M.P., Fayed.M and Ng, H.D. Flapping dynamics of a flexible filament. Physical Review E (2011) in press.

29. Yamaguchi, N., Sekiguchi, T., Yokota, K., Tsujimoto, Y.: Flutter limits and behaviours of a flexible thin sheet in high-speed flow-II: experimental results and predicted behaviours for low mass ratios. J. Fluid Eng. 122 74-83 (2000)

30. Huang, N.E., Shen, Z., Long, S.R., Wu, M.C., Shih, H.H., Zheng, Q., Yen, N.C., Tung, C.C., Liu., H.H: The Empirical Mode Decomposition and Hilbert Spectrum for Nonlinear and Nonstationary Time Series Analysis. P Roy Soc Lond A, 454 903-995 (1998)

31. Lai, D., Chen, G.: Statistical analysis of Lyapunov exponents from time series: A Jacobian approach. Math Comput. Model 27 1-9 (1998)

32. Grebogi, C., Ott, E., Pelikan, S., Yorke, J.A.: Strange attractors that are non chaotic. Physica D, $13261-$ 268 (1984)

33. Ditto, W.L., Spano, M.L, Savage, H.T., Rauseo, S.N., Heagy, J., Ott, E.: Experimental observation of a stranger nonchaotic attractor. Phys. Rev. Lett._65_553 (1990)

34. Nishikawa, T., Kaneko, K.: Fractalization of a torus as a strange nonchaotic attractor. Phys. Rev. E 54, 6114-6124 (1996)

35. Bergé, P., Pomeau, Y., Vidal, C.: Order Within Chaos Hermann (1984).

36. Nayfeh, A.H., and B. Balachandran. Applied Nonlinear Dynamics. John Wiley \& Sons, New York. (1995). 


\section{Figures}

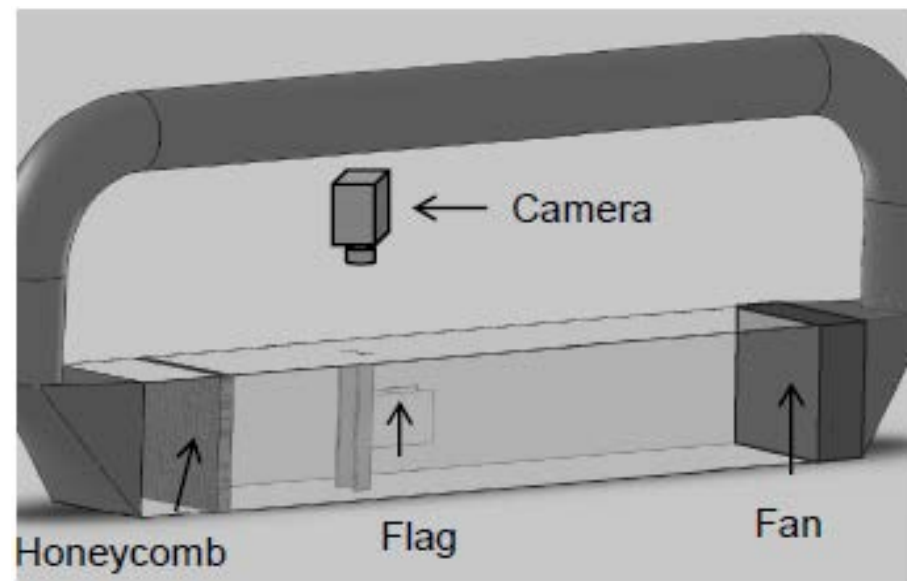

Fig.1: Experimental setup

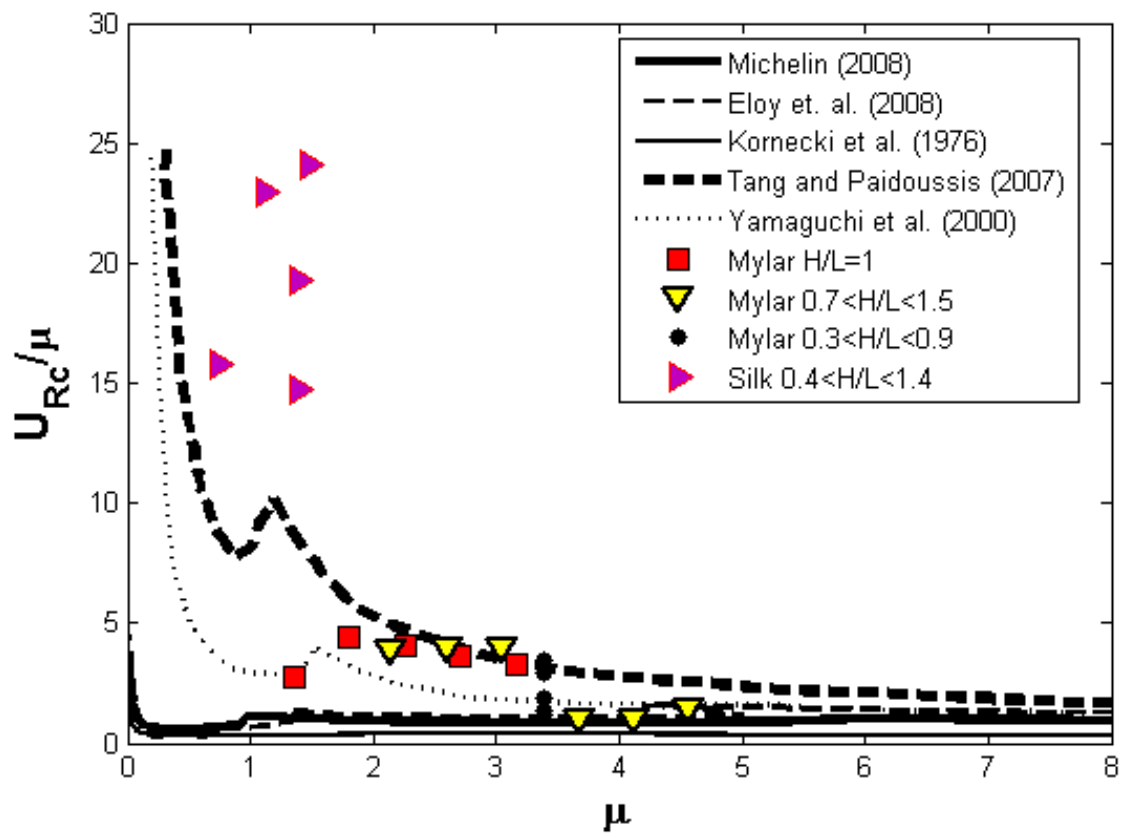

Fig.2: Normalized critical velocity $\mathrm{U}_{\mathrm{Rc}}$ versus the mass ratio $\mu$; here $U_{R c}=U L \sqrt{\frac{\rho_{m} h}{D}}$ and $\mu=\rho_{f} L / \rho_{m} h$ 


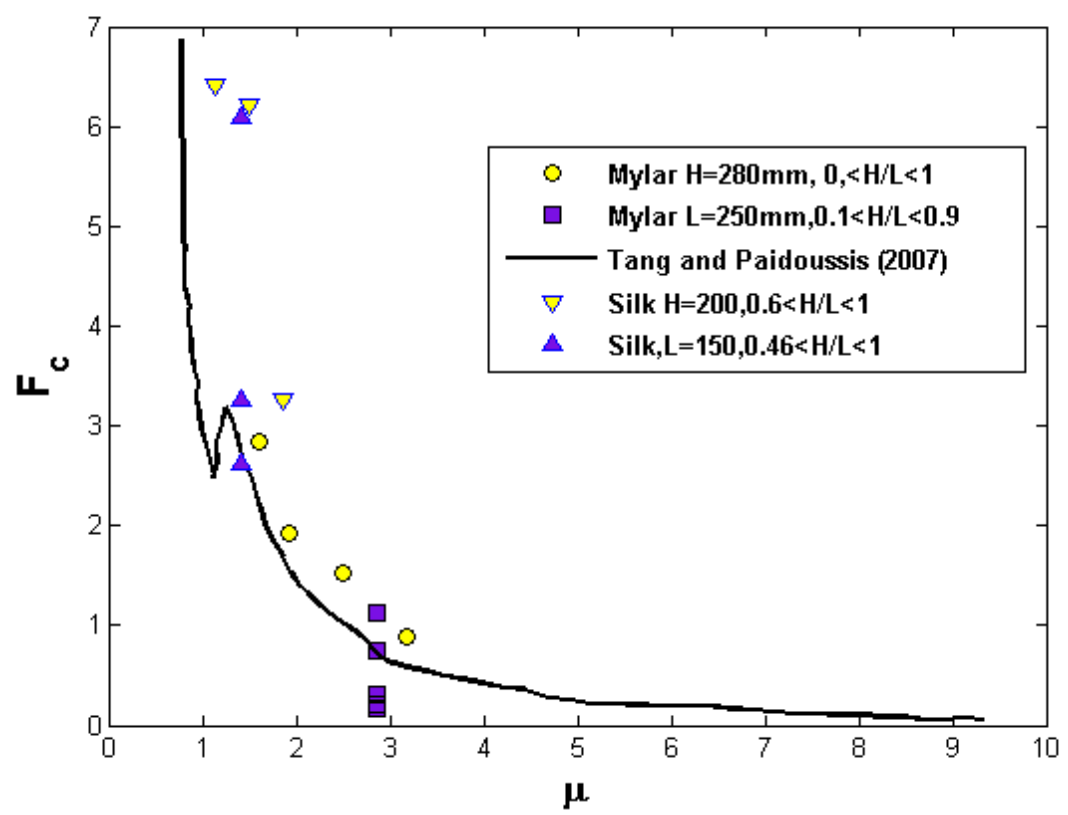

Fig.3: Normalised frequency at onset of flapping $F_{c}=\left(\rho_{m} h\right)^{5 / 2} /\left(\rho_{f} D^{1 / 2}\right) f_{c}$ versus the mass ratio $\mu ; f_{c}$ is the estimated flapping frequency at its onset; $\rho_{m}$ and $\rho_{f}$ are the flag and fluid density, respectively; $D$ is the flexural rigidity of the flag. 

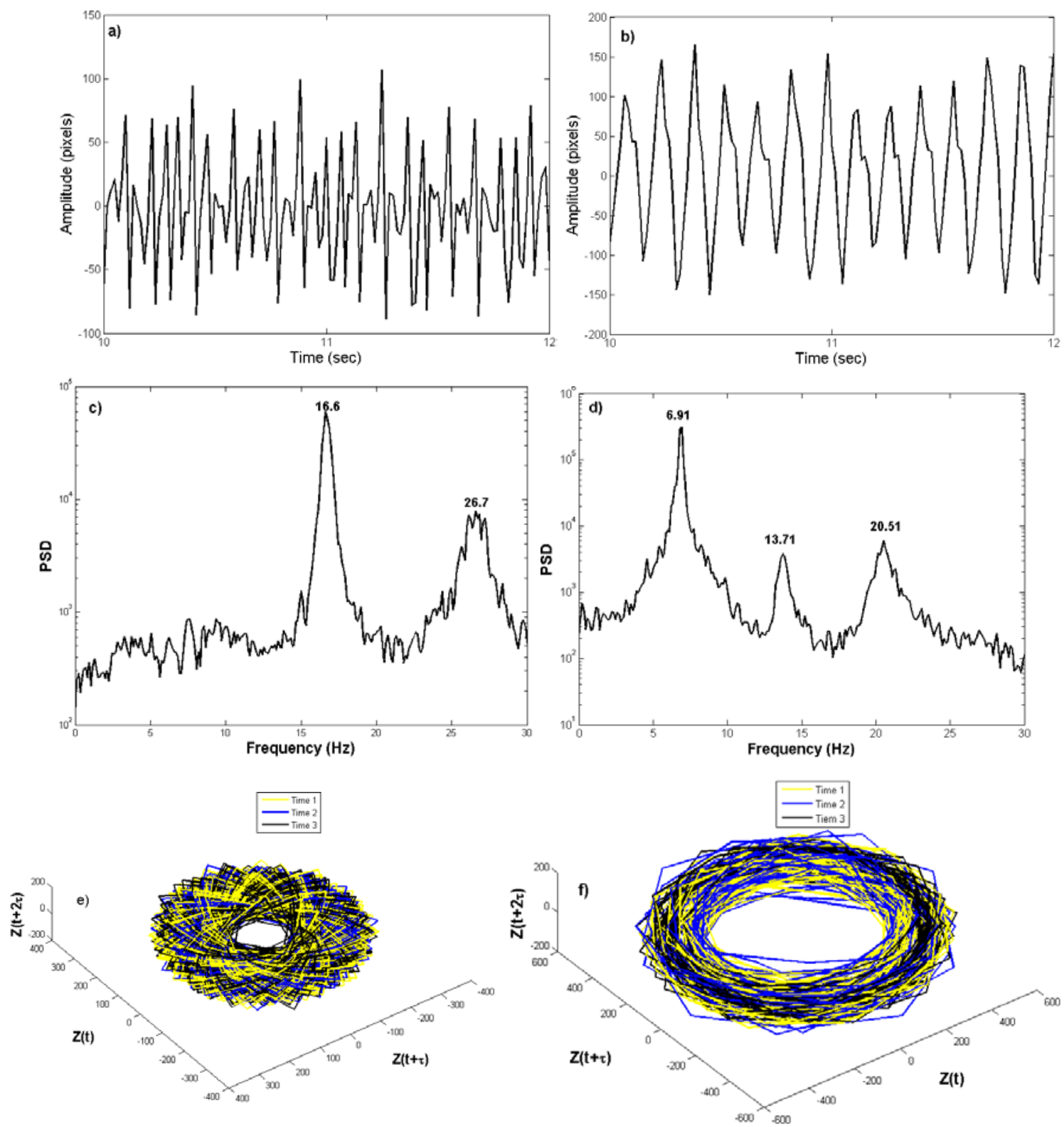

25 

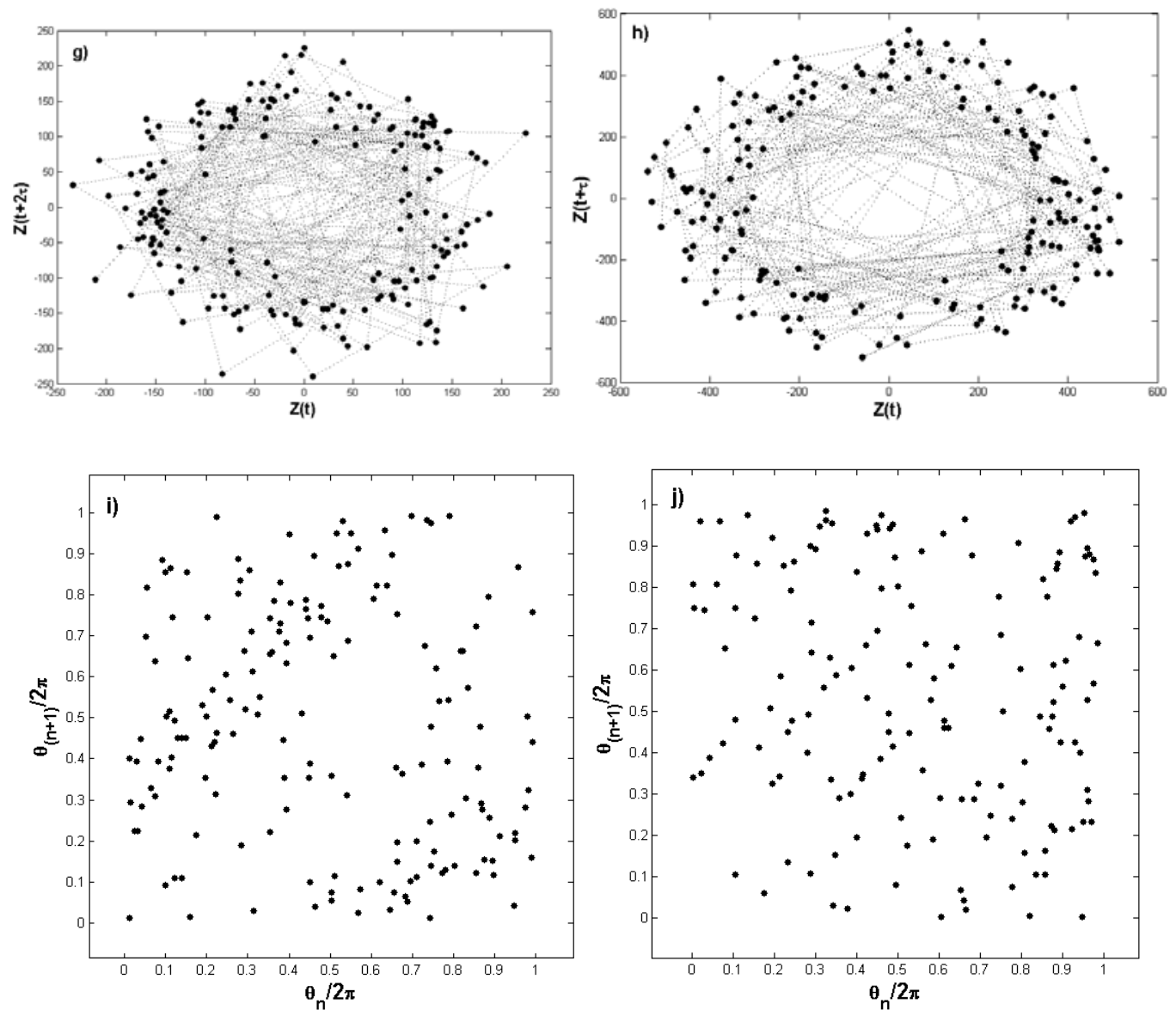

Fig.4. Flapping dynamics for short Mylar and silk flags. Aspect ratio and flow velocity for Mylar flag are $H / L=2$ and $5.76 \mathrm{~m} / \mathrm{s}$ respectively. Aspect ratio and flow velocity for silk flag are $H / L=1.67$ and $3 \mathrm{~m} / \mathrm{s}$, respectively. (a) and (b) Transverse displacement of a point at the top edge of the flag located at $3 / 4$ from the leading edge for Mylar and silk flags, respectively ; (c) and (d) corresponding power spectrum; (e) and (f) reconstructed attractors for Mylar and silk flags, respectively; (g) and (h) corresponding Poincaré sections; (i) and (j) corresponding circle maps. 

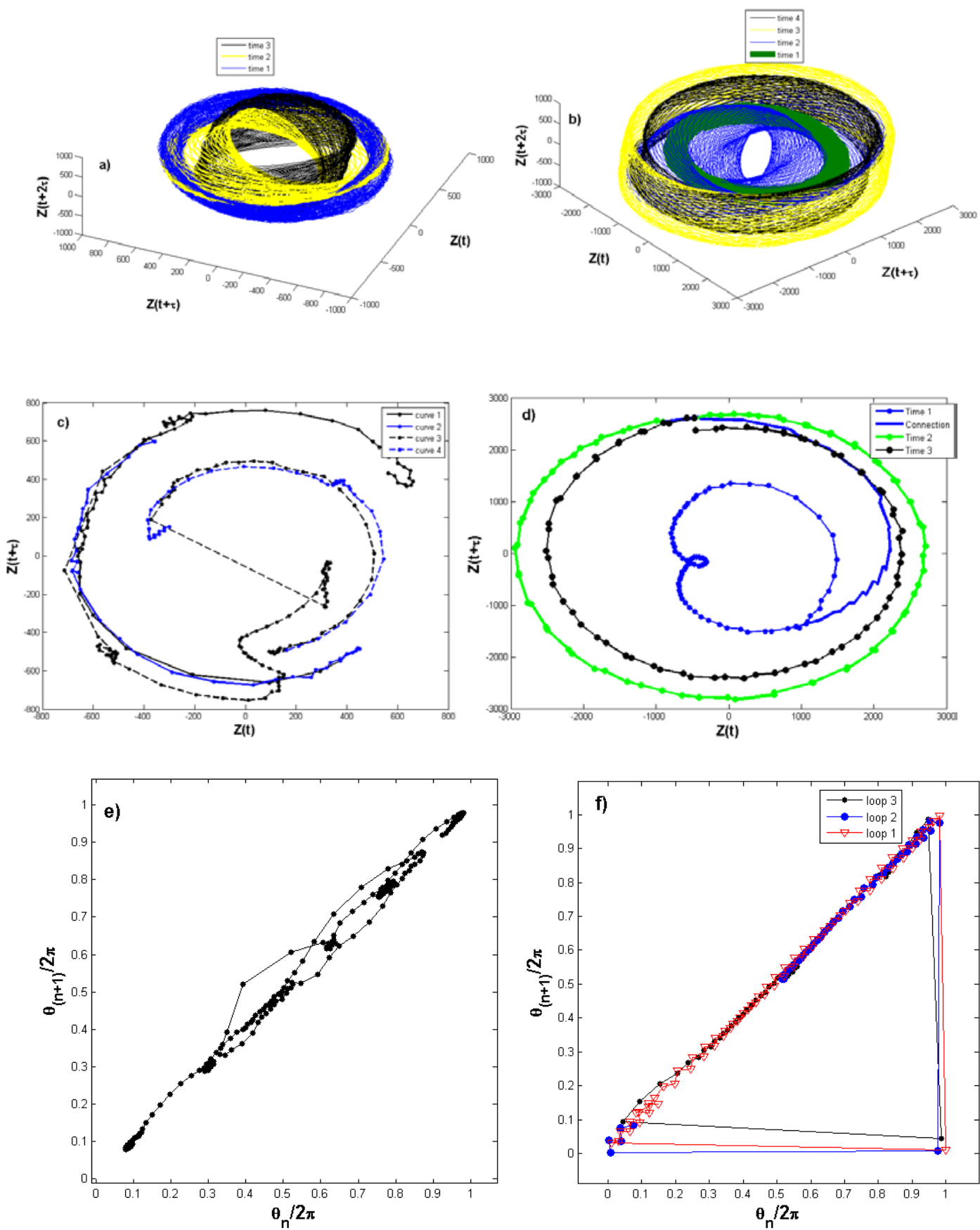

Fig.5. Flapping dynamics for Mylar and silk flags with aspect ratio equal to 1 . The flow velocity for the Mylar and silk flags is $2.45 \mathrm{~m} / \mathrm{s}$ and $1.84 \mathrm{~m} / \mathrm{s}$, respectively. (a) and (b) Reconstructed attractors for Mylar and silk flags, respectively; (c) and (d) corresponding Poincaré sections, (e) and (f) corresponding circle maps. 

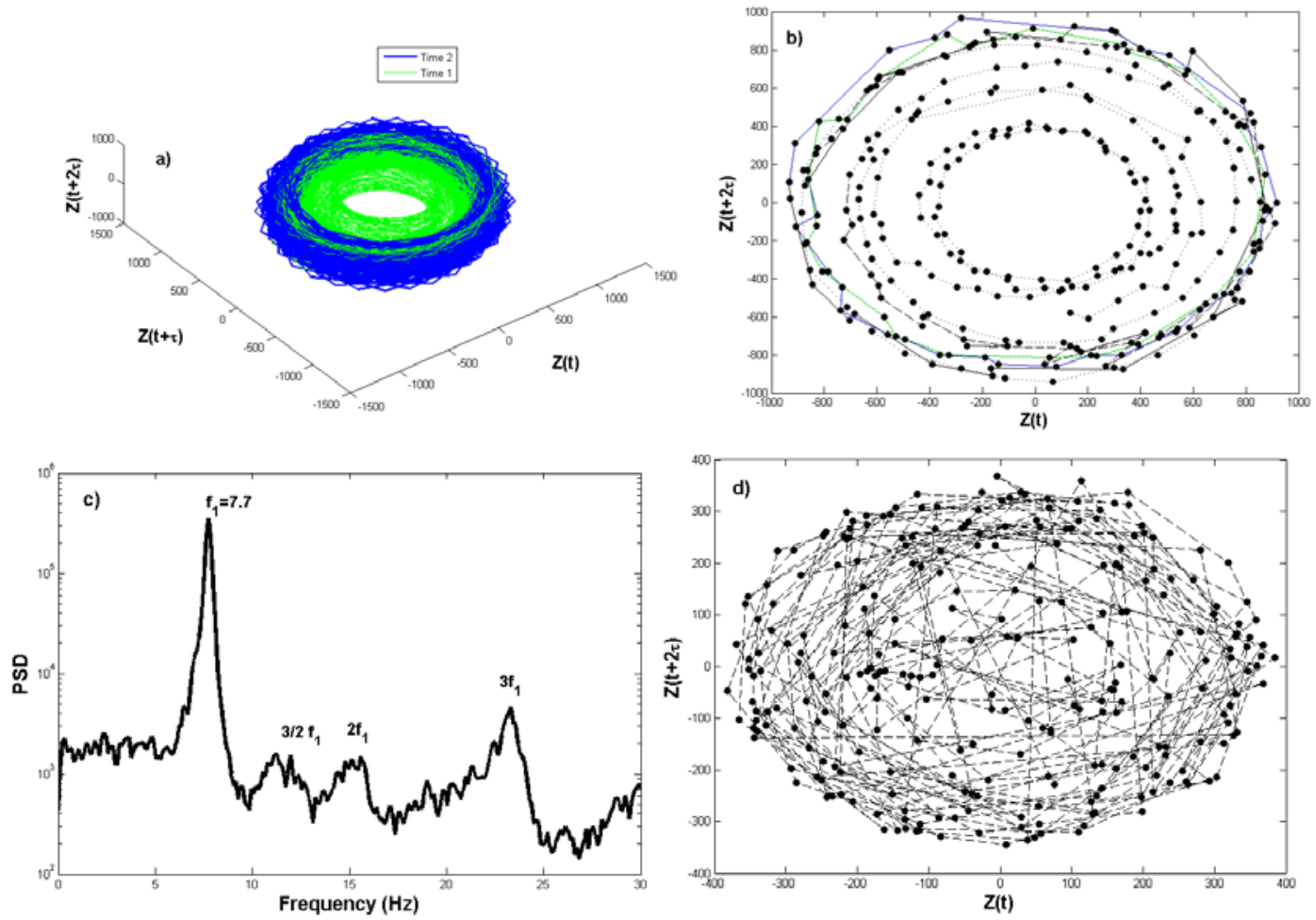

Fig.6. Flapping dynamics for the silk flag with aspect ratio equal to one. For flow speed equal to $2.82 \mathrm{~m} / \mathrm{s}$ : (a) Reconstructed attractors, (b) Poincaré section, (c) power spectrum. (d) Circle map of the flapping dynamics when the flow speed had been increased to $3.3 \mathrm{~m} / \mathrm{s}$. 

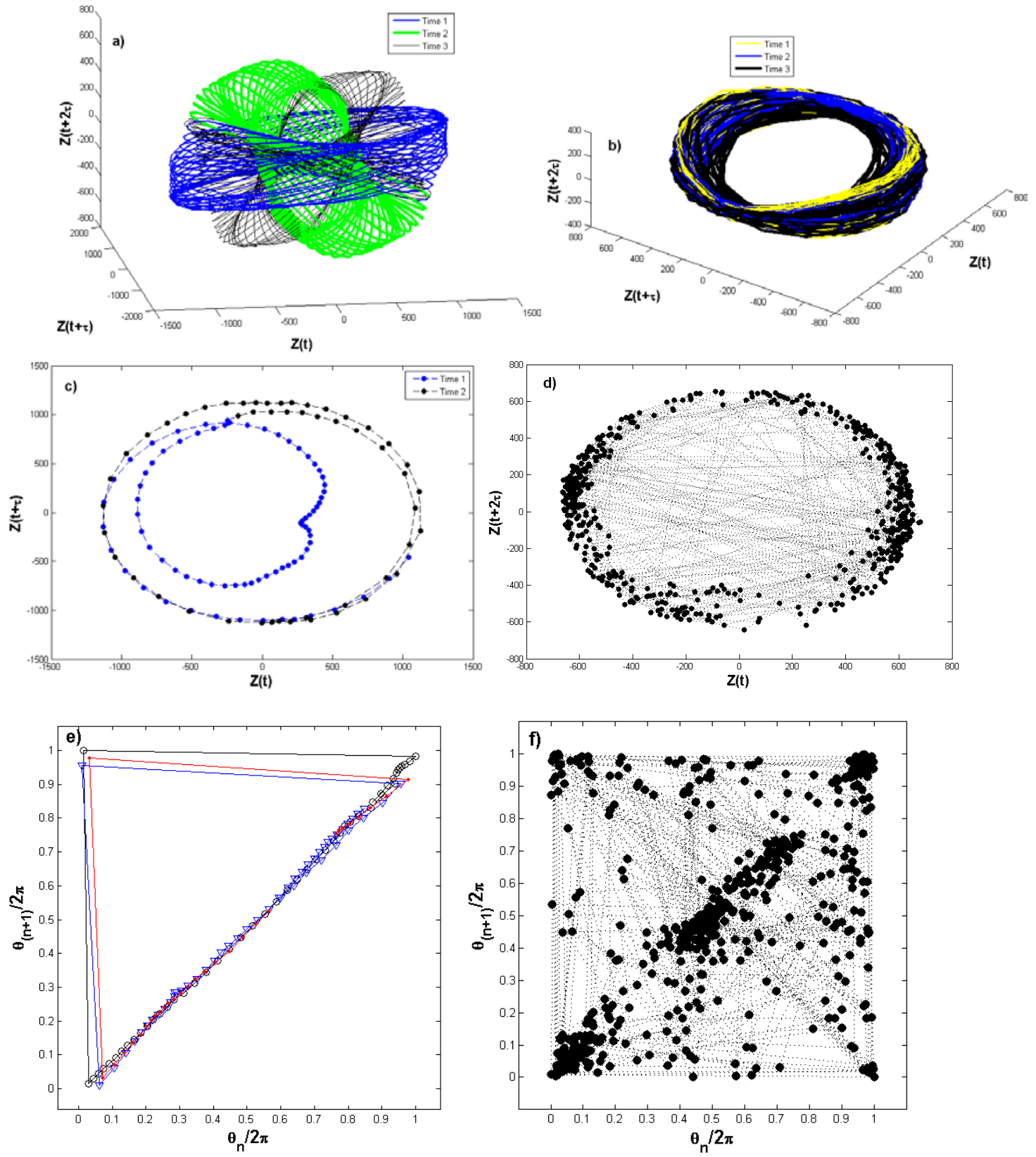

Fig.7. Flapping dynamics for long (a,c,e) Mylar and (b,d,f) silk flags. Aspect ratio and flow velocity for the Mylar flag are $H / L=0.1$ and $1.84 \mathrm{~m} / \mathrm{s}$, respectively. Aspect ratio and flow velocity for silk flag are $H / L=$ 0.46 and $1.75 \mathrm{~m} / \mathrm{s}$, respectively. (a) and (b) Reconstructed attractors; (c) and (d) corresponding Poincaré sections; (e) and (f) circle map. 

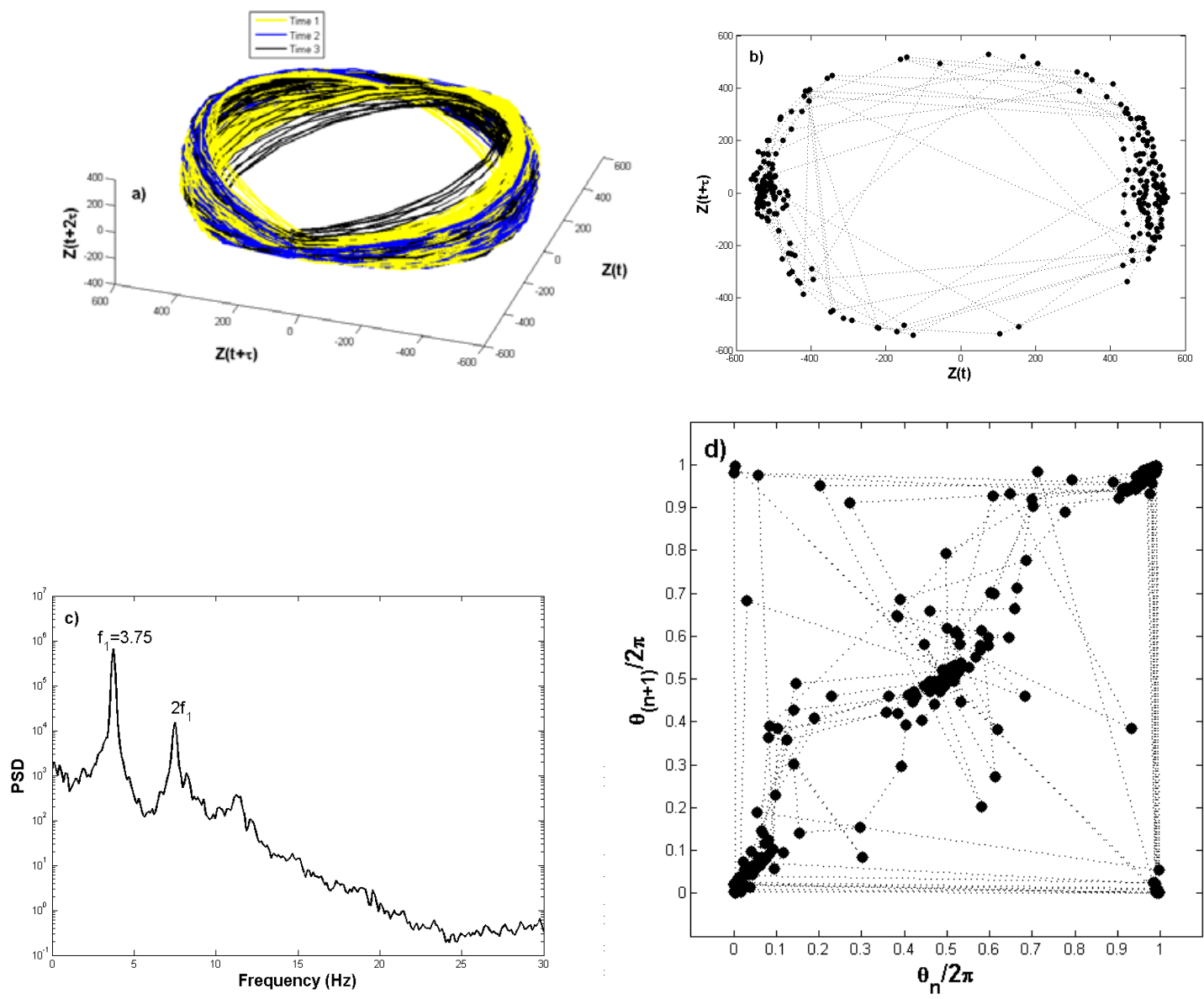

Fig.8. Flapping dynamics for long Mylar flags. Aspect ratio and flow velocity for Mylar flag are $H / L=0.1$ and $3.3 \mathrm{~m} / \mathrm{s}$, respectively. (a) Reconstructed attractors; (b) Poincaré section; (c) power spectrum; (d) circle maps. 

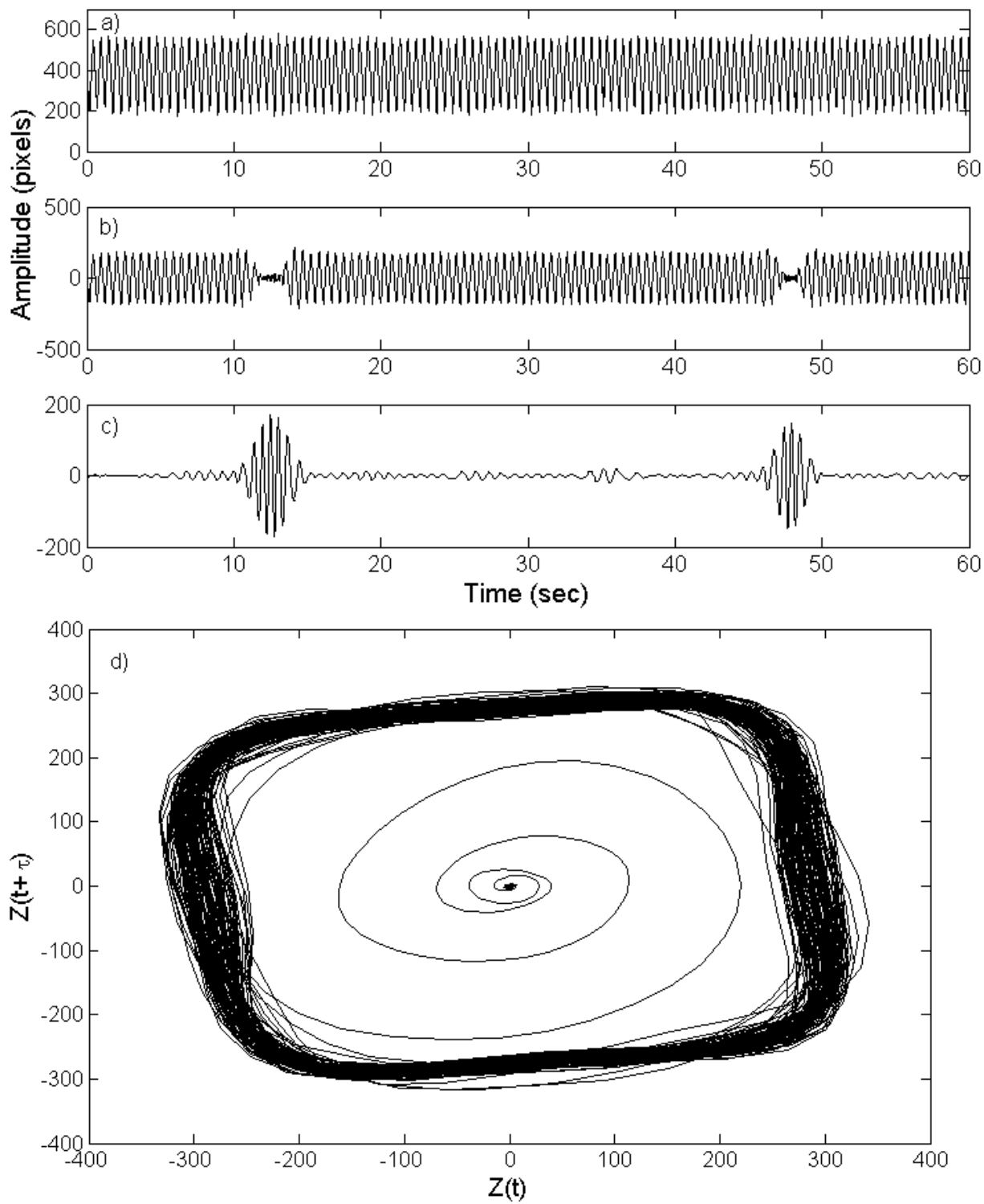

Fig.9. Switching oscillation phenomenon for long Mylar flag $(H / L=0.1)$, velocity $1.84 \mathrm{~m} / \mathrm{s}$, just below the critical flow velocity. (a) Raw time series; (b) and (c) first two empirical mode decompositions; (d) phase space plot. 